\title{
Redução de danos: análise das concepçôes que orientam as práticas no Brasil
}

\author{
I 1 Vilmar Ezequiel dos Santos, ${ }^{2}$ Cássia Baldini Soares, \\ ${ }^{3}$ Célia Maria Sivalli Campos 1
}

Resumo: Este estudo teve como objetivo analisar as concepções que orientam as práticas de Redução de Danos (RD) no Brasil. Selecionaram-se 46 publicações nacionais, de 1994 a 2006, tendo como referência principal a base de dados LILACS. Partindo das categorias centrais objeto (aquilo que se pretende transformar) e sujeito (para quem as ações são direcionadas), a análise mostrou que a RD apresenta-se de maneira bastante heterogênea, com a unidade objeto-sujeito representada por diferentes equações: dependência-dependente; doenças transmissíveisusuários de droga das populações marginalizadas e excluídas; consumo-usuário de drogas; moradores-modo de vida de uma dada "comunidade"; riscos sociais e população em geral; e produção, comércio e consumo de Substâncias Psicoativas (SPA)-classe social. Pode-se concluir que as diferentes concepções de RD expressam tendências que vão se constituindo no Brasil como respostas aos problemas relacionados ao complexo fenômeno do consumo de drogas, e significam o envolvimento de diversas áreas e a ampliação do debate teórico.

> Palavras-chave: redução de danos, drogas de abuso, análise qualitativa.

\author{
${ }^{1}$ Mestre e doutorando, \\ Departamento de Enfermagem \\ em Saúde Coletiva da Escola de \\ Enfermagem da Universidade de \\ São Paulo. Endereço eletrônico: \\ vilmar@usp.br. \\ 2 Profa associada, Departamento \\ de Enfermagem em Saúde \\ Coletiva da Escola de \\ Enfermagem da Universidade de \\ São Paulo. Endereço eletrônico: \\ cassiaso@usp.br. \\ ${ }^{3}$ Prof ${ }^{a} \mathrm{Dr}^{\mathrm{a}}$, Departamento de \\ Enfermagem em Saúde Coletiva \\ da Escola de Enfermagem da \\ Universidade de São Paulo. \\ Endereço eletrônico: celiasiv@ \\ usp.br.
}

Recebido em: 31/08/2009. Aprovado em: 03/05/2010. 


\section{Introdução}

O objeto deste trabalho é a concepção de objeto e sujeito que orienta as práticas de Redução de Danos (RD), recortada a partir da análise da literatura brasileira sobre o tema.

As origens da RD como uma prática voltada para o consumo de substâncias psicoativas podem ser localizadas na Inglaterra, em 1926, com o Relatório de Rolleston, o qual concluía que os usuários em situação de dependência de opiáceos poderiam receber estas drogas sob prescrição de um médico, visando a levar uma vida mais estável e mais útil à sociedade (O'HARE, 1994). Considerava-se que, mesmo diante de um consumo dependente de drogas, esses usuários poderiam diminuir as consequências mais danosas, minimizando os efeitos prejudiciais a sua saúde.

Na década de 80 (STIMSON, 1998; BASTOS, 1998), a RD passou a ser reconhecida como uma estratégia de saúde junto aos usuários de drogas. Em função da implantação de vários programas e práticas e das inovações e resultados obtidos, principalmente no campo da prevenção da Aids, a RD veio a ser difundida e se tornou importante estratégia de saúde pública disseminada por vários países, conforme demonstram alguns autores (BERRIDGE, 1993; REALE, 1997; BASTOS, 1998; FONSECA, 2005).

Desde as primeiras iniciativas, em 1989 na cidade de Santos (MESQUITA, 1998; BUENO, 1998), o Brasil vem se apresentando como um país de forte tradição na implantação de políticas de RD e dessa forma sedia parcela considerável das discussões teóricas que amparam as práticas.

Neste estudo, parte-se do pressuposto de que a RD tem-se apresentado como uma alternativa no campo das práticas de saúde relacionadas aos problemas advindos do consumo de substâncias psicoativas, que resgata aspectos éticos e humanos da relação entre políticas de saúde e consumidores de drogas. No entanto, a $\mathrm{RD}$ tem se revestido de um excesso de pragmatismo, muitas vezes reproduzindo práticas sem demonstrar claramente em que bases teóricas está ancorada. Analisar as publicações brasileiras sobre RD buscando compreender as bases teóricas que a sustentam pode ser um importante instrumento para ampliar o seu alcance, produzindo práticas inovadoras e abrangentes. 


\section{Seleção dos textos}

Para selecionar os textos a serem analisados, procedeu-se, num primeiro momento, a um levantamento bibliográfico, realizado em julho de 2006 na base de dados LILACS (http://www.bireme.br), buscando-se todas as publicaçōes sobre RD no Brasil. Foram excluídos os que apenas tangenciavam as questóes pertinentes a este trabalho e os que se repetiam em termos de conteúdo. Tendo em vista que a produção científica brasileira sobre esse tema é ainda bastante incipiente, não se restringiu o período de busca. Novos textos foram, num segundo momento, incorporados ao conjunto primeiramente levantado, considerando-se sua expressividade na área, bem como sua importância para as políticas públicas brasileiras.

Dessa forma, foram selecionados 46 trabalhos, incluindo capítulos de livros, artigos de revistas indexadas, manuais e documentos oficiais, dissertações de mestrado e teses de doutorado, o que constitui uma amostra considerável e substancialmente importante da bibliografia brasileira em RD.

\section{Análise do material}

Para a leitura dos estudos produzidos no Brasil, utilizou-se o método de análise de conteúdo, conforme descrito por Triviños (1992), que parte da orientação de Bardin (2006), salientando a importância do apoio do referencial teórico e do método dialético na composição das categorias de análise e no desenvolvimento da análise propriamente dita.

Realizou-se primeiramente uma leitura cuidadosa dos trabalhos, visando a obter uma compreensão global dos conteúdos descritos e abstrair a ideia central sobre RD. Em seguida foram selecionados os trechos dos textos que se referiam mais particularmente ao objeto e ao sujeito da $\mathrm{RD}$, sendo recortados e classificados em unidades comparáveis de categorização (BARDIN, 2006) de acordo com as semelhantes formas de concebê-los.

A categorização foi empregada à medida que os trabalhos foram sendo analisados, não sendo portanto definida a priori. $\mathrm{O}$ próprio material analisado conferiu forma e sentido a um conjunto de categorias constituídas para classificar os trabalhos, como refere Bardin (2006) sobre o emprego da categorização para dois processos 
inversos. A análise foi concluída no mês de abril de 2008, por ocasião da defesa da dissertação de mestrado intitulada $O$ objeto/sujeito da redução de danos: uma análise da literatura brasileira da perspectiva da saúde coletiva, defendida na Escola de Enfermagem da Universidade de São Paulo, na qual se baseia o presente artigo.

\section{Resultados}

Dos trabalhos selecionados, $50 \%$ eram oriundos de universidades, $41 \%$ de instituiçôes públicas (30\% ligadas às políticas de prevenção/controle de doenças transmissíveis em geral e de HIV/Aids em particular e 11\% ligadas às políticas de saúde mental) e 9\% advinham de associações, redes e organizações da sociedade civil (gráfico 1). Observa-se que no Brasil há uma crescente assimilação da RD pelo poder público, inicialmente como uma estratégia efetiva no campo das doenças transmissíveis e, nos últimos anos, se expandindo como modelo de política para o campo da saúde mental.

\section{Gráfico 1: Instituições de origem dos trabalhos analisados.}

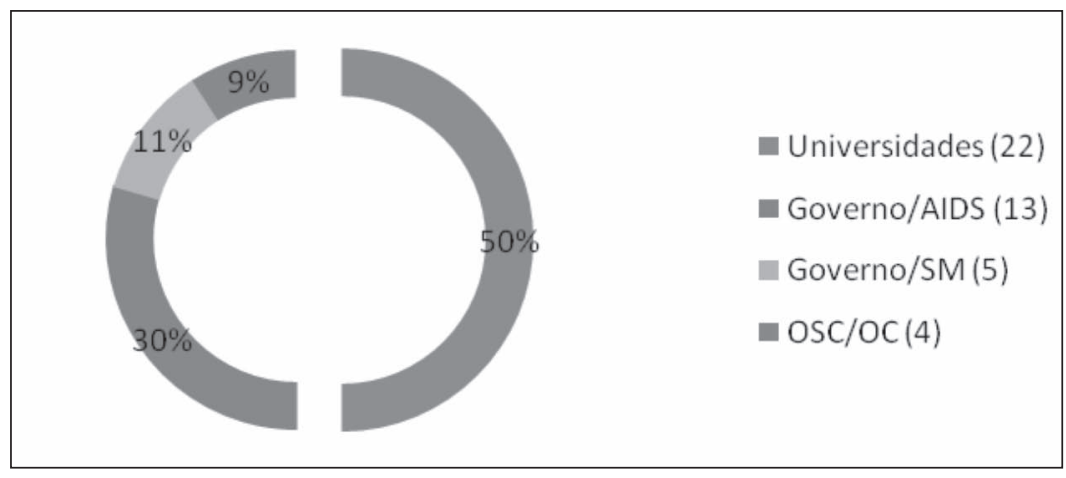

Fonte: Santos (2008).

Em relação ao campo do saber que vem se ocupando das produções em RD, observou-se que $30 \%$ vinham da área da psiquiatria, $20 \%$ da saúde pública e epidemiologia, $20 \%$ da psicologia, $9 \%$ da saúde coletiva, $7 \%$ das ciências sociais, $5 \%$ da antropologia, $2 \%$ do serviço social e $2 \%$ da criminologia crítica, sendo que não foi possível identificar a área do saber em 5\% dos trabalhos (gráfico 2). Nota-se que a área que mais vem se apropriando da produção de conhecimentos e práticas relativas à RD é a psiquiatria. Com a expansão da 
epidemia do HIV/Aids, a saúde pública e a epidemiologia acabam se voltando para esse objeto. Observa-se ainda que outras áreas do saber buscam dar um contorno mais amplo e político para a RD. É o caso da saúde coletiva, das ciências sociais, da criminologia crítica e de algumas vertentes da antropologia.

Gráfico 2: Áreas do conhecimento dos trabalhos analisados

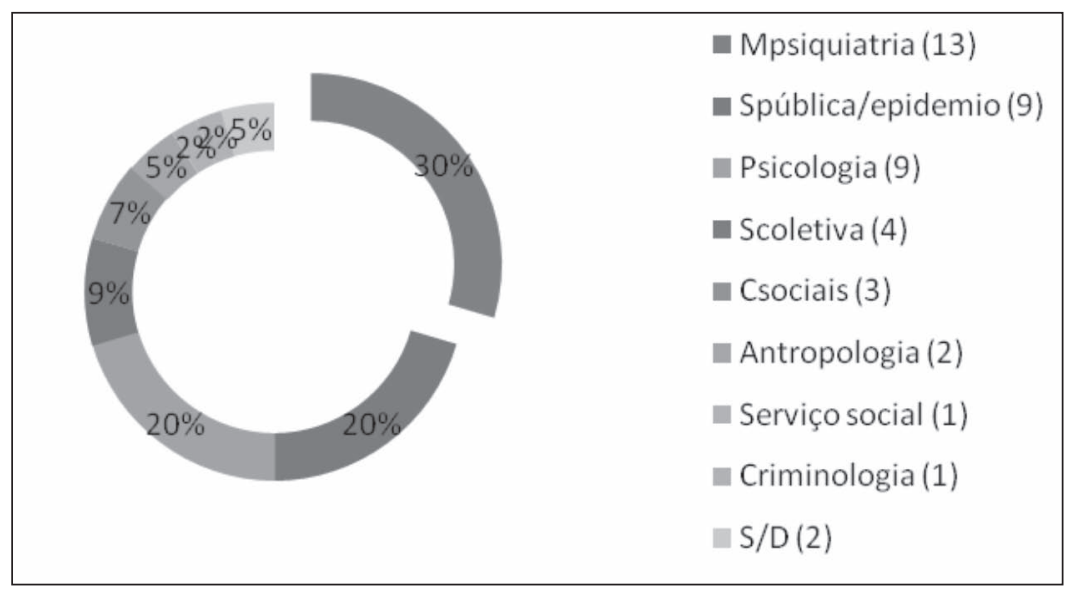

Fonte: Santos (2008).

Quanto ao tipo de publicação observa-se que 43\% eram capítulos de livros, $39 \%$ artigos de revistas indexadas, 9\% manuais, 4,5\% eram dissertaçōes e 4,5\% teses (gráfico 3). A diversidade quanto às áreas e aos tipos de publicação acabou por oferecer uma amplitude maior de conteúdos para análise.

Gráfico 3: Tipo de publicação dos trabalhos selecionados

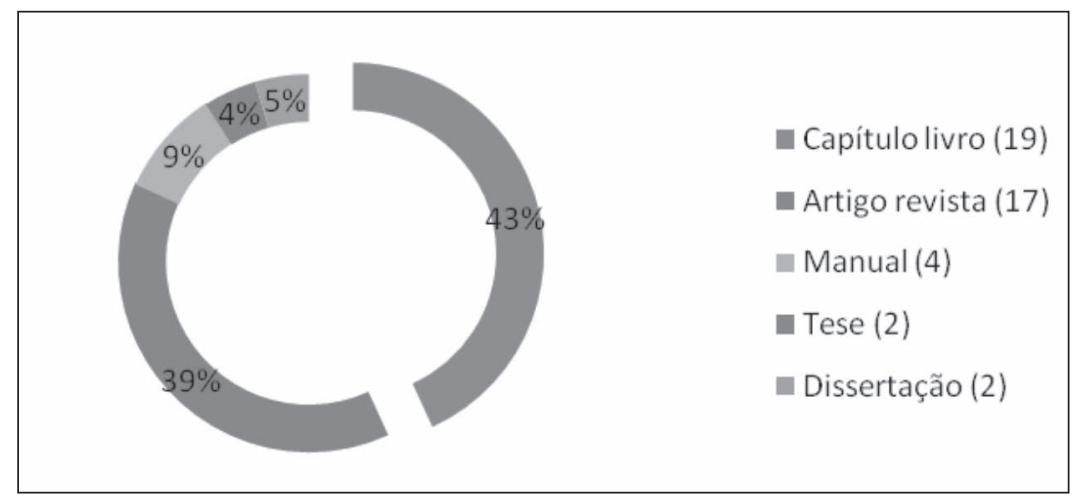

Fonte: Santos (2008). 
A análise dos trabalhos buscou apreender em cada produção em particular aquilo que estava sendo tomado como objeto/sujeito da RD. Para melhor compreender as diferenças encontradas, optou-se por construir uma classificação em subcategorias menores, que supostamente exporiam a unidade objeto (o que se deseja conhecer e transformar)-sujeito (quem se deseja conhecer e transformar) de maneira mais clara. Partiu-se da compreensão de que os diversos recortes de objeto estariam ancorados em distintas concepçōes de saúde e do complexo sistema de produção, distribuição e consumo de substâncias psicoativas na contemporaneidade, classificando-se os trabalhos em função do recorte do objeto/sujeito da RD conforme sintetizado a seguir. Ainda cabe salientar que um mesmo trabalho pode estar classificado em mais de uma categoria, denotando em algumas situações mudanças ou ampliações na forma de conceber o objeto/ sujeito entre os autores e/ou entre distintos trabalhos de um mesmo autor.

\section{Quando o objeto/sujeito se encontra} na unidade dependência-dependente

Sete trabalhos (ANDRADE, 1998; GUERRA et al., 2003; RIBEIRO; LARANJEIRA, 2003; CARLINI, 2003; DIAS et al., 2003; CRUZ et al., 2003; CARLINI-MARLATT et al., 2003) recortam o objeto da RD pelo ângulo da dependência, propondo que a finalidade das ações seja a abstinência de qualquer consumo de drogas. Parte-se do pressuposto que o consumo caminha sempre no sentido de um uso dependente ou pelo menos problemático e por isso seria necessário evitar que um primeiro uso ocorra. Considerando que nenhuma forma de consumo é tolerada, a RD é tomada tão somente como uma estratégia para se chegar aos usuários que não aceitam os tratamentos voltados para a abstinência, sendo denominada por alguns autores de prevenção secundária ou terciária. Vem da psiquiatria contribuição significativa dessa concepção. Os sujeitos são concebidos como passivos diante do uso, frágeis, incapazes de se adaptar socialmente às normas e regras sociais e nesse sentido ficam subjugados e cooptados pelo poder da droga, não conseguem escapar do caminho determinista de, iniciado o uso, tornar-se dependente.

Nesse sentido, os conceitos e estratégias parecem se orientar para afastar a droga do indivíduo ou o indivíduo da droga, pois o entendimento é que essa relação sempre vai ter como fim último a dependência. Essa forma de conceber o 
sujeito tem servido de justificativa para a manutenção de respostas conservadoras e autoritárias (BRITES, 2006, p. 47) oferecendo suporte para a criminalização do consumo de um conjunto de substâncias psicoativas, mas que também tem sido suporte para as políticas em relação às drogas lícitas.

\section{Quando o objeto/sujeito é a unidade doenças transmissíveis-usuários de droga das populaçóes marginalizadas e excluídas}

Vinte e dois trabalhos (CARLINI, 2003; DIAS et al., 2003; CRUZ et al., 2003; CARLINI-MARLATT et al., 2003; FERNANDEZ, 1994; GRANJEIRO et al., 1998; MESQUITA; RIBEIRO, 1998; MARQUES; DONEDA, 1999; BRASIL, 2001a; BRASIL, 2001b; KRAL et al., 2001; HACKER et al., 2001; TELLES et al., 2001; ANDRADE et al., 2001; KUCHENBECKER; SUDBRACK, 2001; FIGUEIREDO; GREDORI, 2002; PAES, 2002; TRIGUEIROS; BUENO, 2003; MARTINS, 2003; GUERRA et al., 2003; MOREIRA; SILVEIRA, 2003; MALBEGIER et al., 2003) compreendem que o objeto da RD é o risco de doenças transmissíveis, com ênfase no HIV/Aids, e também outros agravos à saúde que acometem os usuários de drogas, especialmente aqueles que fazem parte de grupos marginalizados ou excluídos. Essa perspectiva está relacionada com as primeiras estratégias de RD implantadas no Brasil, que a princípio estavam voltadas unicamente para a prevenção de hepatite e Aids entre usuários de droga injetável. Nessa lógica predominam as noçôes de risco e/ou de vulnerabilidade para doenças e outros agravos à saúde e o conceito de dano relacionado à transmissão do HIV. Predomina a concepção de que é a condição de marginalização e exclusão que expõe os usuários a maiores riscos e agravos, assim a meta é a prevenção de doenças transmissíveis e a aproximação dos usuários às ações de saúde.

Nesse contexto, o sujeito é o usuário de drogas proveniente de populações marginalizadas e excluídas. Parte-se do pressuposto que, em função de preconceitos e por temor, geralmente os sujeitos são refratários ou reticentes a procurar espontaneamente os serviços de saúde. A RD apresenta-se como uma estratégia para a formação de vínculos através do oferecimento de insumos e de orientaçōes para prevenção de doenças transmissíveis e outros agravos à saúde. Ao viabilizar a atenção à saúde para usuários de drogas ilícitas, a RD acaba sendo porta-voz do reconhecimento de direitos sociais desse grupo em particular. 


\section{Quando o objeto/sujeito tem a unidade}

\section{na equação consumo-usuário de drogas}

Nesse recorte, as concepções orientadoras das práticas expressas em 17 trabalhos (REALE, 1997; FONSECA, 2005; CRUZ et al., 2003; FIGUEIREDO; GREDORI, 2002; MARTINS, 2003; MOREIRA; SILVEIRA, 2003; BASTOS, 2003; STRONACH, 2003; RIBEIRO; LARANJEIRA, 2003; MACRAE; GORGULHO, 2003; FERREIRA, 2003; MOREIRA et al., 2006; SODELLI, 2006; SILVEIRA et al., 2003; CAMPOS; SIQUEIRA, 2003; VELOSO et al., 2004; NARDI; RIGONI, 2005) podem ser resumidas da seguinte forma: muitos usuários não conseguem abandonar por completo o uso e outros desejam continuar usando, por vezes, expondo-se a riscos e prejuízos; a busca pelas substâncias psicoativas serve a diversos propósitos humanos, ou seja, as drogas sempre foram objeto de interesse dos homens; a droga não é considerada o agente determinante e a relação que se estabelece entre o sujeito e a substância psicoativa deixa de ser necessariamente de dependência, sendo considerados outros tipos e repercussões do uso. A alteração da psicoatividade é compreendida como possibilidade de aplacar as angústias geradas pelas condições de existência. Assim, a meta da RD é construir estratégias e possibilidades de diminuição dos danos e riscos relacionados ao consumo prejudicial ou de risco; a relação de dependência pode ser minimizada. As estratégias de reduzir riscos e danos podem ser múltiplas dependendo da substância, do tipo de uso, das circunstâncias em que ele ocorre e outras variáveis do contexto sócio-ambiental e cultural.

O usuário de drogas deixa de ser considerado passivo diante do consumo e com capacidade para adotar formas de consumo menos prejudiciais. A droga não é considerada o agente determinante e a relação que se estabelece não é necessariamente de dependência. Nesse sentido a lógica se inverte, pois, se no primeiro caso as açóes e políticas se voltam para a substância, aqui elas se voltam para o usuário, para orientá-lo, informá-lo e criar condições para desenvolver autonomia visando ao autocuidado.

O resultado desta nova equação pode significar um excesso de responsabilização individual, tanto pelo sucesso como pelo fracasso das iniciativas por parte dos usuários. Estes ganham na condição em si de usuários de drogas sua "identidade", perdendo quase que completamente sua referência como sujeito social. Essa tendência de criar identidades particularizadas em uma dada condição vem 
sendo apontada na contemporaneidade (WOOD, 1999). O consumo de

drogas da forma como se apresenta na atualidade não seria um encontro com uma vulnerabilidade própria e sempre presente no ser humano, mas estaria relacionado diretamente com as transformações das sociedades e dos novos processos subjetivos constituídos na pós-modernidade (BIRMAN, 2006).

Esse novo cenário no qual se constituem novas subjetividades tem sua base alicerçada no modo de produção capitalista. Carneiro (2002) fornece uma compreensão das formas atuais de consumo compulsivo como consequência da reprodução ampliada do capital e da transformação dos produtos em fetiches da forma-mercadoria.

\section{Quando o objeto/sujeito da redução de danos está} alicerçado no modo de viver de uma dada "comunidade"

Em oito trabalhos (FONSECA, 2005; BASTOS, 2003; MOREIRA et al., 2006; SILVEIRA et al., 2003; CAMPOS; SIQUEIRA, 2003; TAVARES-DELIMA, 2003; DELGADO et al., 2003; BRASIL, 2004), o objeto da RD pode ser interpretado como sendo o modo de viver em uma dada comunidade. A finalidade das ações é a promoção de estilos de vida saudável, a melhoria da qualidade de vida e a promoção da saúde, ampliando-se, portanto, os objetivos a serem alcançados, seja por referência à prevenção de doenças transmissíveis e agravos à saúde individual, seja por referência à redução de prejuízos relacionados ao consumo de drogas. Essa perspectiva se fundamenta na concepção que correlaciona diversos fatores no desencadeamento e manutenção do consumo prejudicial de drogas. Esses fatores dizem respeito às particularidades da droga, às características do sujeito e as inter-relações com o contexto sociocultural. No entanto, conforme refere Soares (2007), o contexto só é referido como uma variável que quando muito se soma às demais ou para proteger o sujeito da aquisição do hábito do consumo ou oferecendo riscos que propiciam o uso e a progressão para a dependência.

Os fatores de risco e de proteção são utilizados para planejar intervenções preventivas. As práticas em saúde ficam assim restritas a alertar os grupos sociais para os riscos e perigos do consumo e a orientar como estes devem se proteger para não consumir drogas ou, caso consumam, como devem se comportar para que o consumo não evolua para níveis mais elevados até a dependência. 
A promoção da saúde muitas vezes se restringe a propor alternativas para melhorar aspectos da vida que guardariam relação com os danos e riscos relacionados ao consumo prejudicial de drogas. Ao mesmo tempo em que incorpora elementos sociais à compreensão do fenômeno do consumo de drogas, pode ficar restrita aos manuais de bom comportamento. A promoção da saúde também pode servir simplesmente para atestar o controle de grupos dominantes sobre os indivíduos e comunidades (STOTZ; ARAÚJO, 2004) quando se reveste de saberes puramente técnico-científicos visando a harmonia social e aprendizado de boas práticas de saúde e estilos de vida saudável. Permanece o pressuposto de que a comunidade é a principal responsável por produzir mudanças nos seus estilos de vida marginalizando temas como o conflito de classes e a relação capitalismo e saúde, despolitizando o debate (SOARES, 2007, p. 8).

Neste recorte, o sujeito é a comunidade de indivíduos, geralmente considerados capazes de escolhas pessoais e estimulados a desenvolver autonomia para o cuidado consigo. Essa compreensão de sujeito supera, de um lado, a perspectiva de passividade oriunda da vertente que atribui supremacia à substância e, de outro, a perspectiva que privilegia o sujeito individual-usuário de droga, considerando-o também como sujeito plural. Porém, os indivíduos são considerados como se estivessem submetidos a processos idênticos de desgaste e fortalecimento. Assim, muitas vezes, parece tratar-se de um sujeito descontextualizado das suas condições históricas e materiais de existência, apenas referido a uma ideia de comunidade homogênea, unida, estável e sem contradiçôes (CALIPO, 2002, p. 109). A dimensão política e problematizadora da participação dos grupos sociais na resolução de conflitos fica secundarizada ou é relegada completamente (STOTZ; ARAÚJO, 2004).

\section{Quando o objeto/sujeito é representado pela unidade entre os riscos sociais e a população em geral}

Em seis trabalhos observa-se que algumas compreensões (GUERRA et al., 2003; MOREIRA; SILVEIRA, 2003; STRONACH, 2003; DELGADO et al., 2003; FRANCH, 2003; MELCOP, 2003) que fundamentam a RD têm exposto um objeto numa dimensão mais ampla que os riscos individuais, preocupando-se então com os riscos sociais. Nesse caso, a finalidade das ações se coloca na esfera de diminuir riscos e agravos sociais na população de uma maneira geral. Isso seria feito 
através de um conjunto de modificações: nos ambientes de consumo de drogas lícitas, no tipo de oferta das substâncias, na adoção de cuidados posteriores ao consumo, em mudanças nas legislaçooes, na restrição de venda em determinadas situações, na responsabilização por dirigir sob o efeito de bebidas alcoólicas, entre outras. A meta central é a diminuição de agravos sociais relacionados ao consumo prejudicial de drogas, geralmente lícitas, que colocam em risco a vida de consumidores e da coletividade, como no caso de acidentes de trânsito, de situações de violência, de consumos de "risco" em ambientes de festa, entre outros.

A concepção de saúde que orienta essa vertente é multifatorial, dando ênfase às formas de controle social sobre as instituições, sujeitos, contextos e situações em que o consumo ocorre, e à proposição de formas moderadas de consumo. Neste recorte, o sujeito é a população em geral, considerada como potencial consumidor de substâncias psicoativas, e cujo consumo pode gerar além de agravos pessoais, problemas sociais. As medidas não são voltadas somente para consumidores, mas também para instituições, como, por exemplo, estabelecimentos nas estradas que vendem álcool, locais de festas com condições adequadas e mais seguras, entre outras. Assim envolvem também a responsabilidade de instituições e do Estado.

\section{Quando objeto/sujeito é exposto pela unidade} produção-comércio-consumo de SPA sendo o sujeito a classe social Observou-se que, em 22 trabalhos, (REALE, 1997; FONSECA, 2005; CRUZ et al., 2003; CARLINI-MARLATT et al., 2003; FERNANDEZ, 1994; FIGUEIREDO; GREDORI, 2002; MARTINS, 2003; MOREIRA; SILVEIRA, 2003; BASTOS, 2003; MACRAE; GORGULHO, 2003; MOREIRA et al., 2006; SODELLI, 2006; SILVEIRA et al., 2003; CAMPOS; SIQUEIRA, 2003; TAVARES-DE-LIMA, 2003; DELGADO et al., 2003; FRANCH, 2003; KARAM, 2003; GORGULHO, 2003; SOARES; CAMPOS, 2004; CANOLETTI; SOARES, 2005; SOARES, 1997), o consumo contemporâneo de drogas e, nesse sentido, a RD, se fundamenta em análises mais gerais, que relacionam esse fenômeno ao sistema complexo - estrutura e dinâmica do modo de produção - que envolve as substâncias psicoativas, contextualizando-o historicamente. Sobressai um conjunto de saberes críticos em relação às formas de compreensão atuais do consumo de drogas e constituições de políticas e respostas sociais para os problemas relacionados. 
Em seis trabalhos, o sujeito é tomado como sendo o coletivo, (FERNANDEZ, 1994; FIGUEIREDO; GREDORI, 2002; SILVEIRA et al., 2003; DELGADO et al., 2003; FRANCH, 2003; SOARES, 1997) dividido em classes ou grupos sociais (LACAZ, 2001), portanto heterogêneo, submetido a condiçôes desiguais de reprodução social. A RD é considerada um conjunto de saberes e práticas que parte de uma compreensão estrutural do complexo sistema de produção, distribuição e consumo de substâncias psicoativas, tal como se apresenta na contemporaneidade, para propor uma ação menos instrumental e mais emancipatória (SOARES, 2007).

Observou-se que em 13 trabalhos (FONSECA, 2005; CRUZ et al., 2003; FERNANDEZ, 1994; MARTINS, 2003; MOREIRA; SILVEIRA, 2003; MACRAE; GORGULHO, 2003; MOREIRA et al., 2006; SODELLI, 2006; CAMPOS; SIQUEIRA, 2003; TAVARES-DE-LIMA, 2003; DELGADO et al., 2003; KARAM, 2003; SOARES, 1997), os autores criticam a política de criminalização do consumo, que, por um lado, não atinge o objetivo de diminuir a procura por drogas e, por outro, gera consequências de exposição a riscos físicos, psíquicos e sociais aos consumidores especialmente quando estes se deparam com o mundo do tráfico e das ações da polícia. Alguns desses estudos contribuem para compreender o impacto do estigma, na configuração da identidade do usuário de drogas. Outros revelam a contradição das políticas proibicionistas: a criminalização, por um lado, objetiva acabar com os problemas sociais e de saúde relacionados ao consumo das drogas ilícitas, por outro, propicia a criação e a proliferação do narcotráfico e as medidas jurídicas acabam por agravar esses problemas (RODRIGUES, 2003).

Alguns trabalhos, que partem dos fundamentos da saúde coletiva para compreender o consumo de substâncias psicoativas, resgatam as transformaçōes que estão ocorrendo na sociedade contemporânea. $\mathrm{O}$ crescimento do consumo em grande escala ocorre de maneira cada vez mais precoce, está associado a problemas de saúde, violência e mortes por causas externas, entre outros. Essa particularidade, presente em nove trabalhos analisados (MARTINS, 2003; BASTOS, 2003; MOREIRA et al., 2006; TAVARES-DE-LIMA, 2003; DELGADO et al., 2003; FRANCH, 2003; KARAM, 2003; GORGULHO, 2003; CANOLETTI; SOARES, 2005), seria resultado da transformação da droga em mercadoria que responde a novas necessidades (CARNEIRO, 2002), cujas características 
são: produção em escala crescente e diversificada; disseminação por todo o planeta; facilitação do acesso e das tecnologias de marketing; arregimentação de consumidores por uma superindústria lícita e pelo narcotráfico.

A partir da Saúde Coletiva, é possível observar a preocupação em compreender a complexidade que cerca o fenômeno das substâncias psicoativas na sociedade contemporânea, e a constituição da $\mathrm{RD}$, como um novo paradigma, requer um arcabouço teórico-metodológico amplo e interdisciplinar que inclua os saberes de diversas áreas para que não fique restrita ao excesso de pragmatismo e a compreensões desvinculadas e desconectadas da totalidade social.

Nesse sentido, a educação, conforme referido em quatro trabalhos (SODELLI, 2006; TAVARES-DE-LIMA, 2006; CANOLETTI; SOARES, 2005; SOARES, 1997), também se apresenta como um recurso de fundamental importância desde que alicerçada em ferramentas teóricas e práticas que permitam a transformação da realidade social. A partir de uma compreensão mais global e crítica sobre a realidade histórica e social, os sujeitos tendem a compreender as raízes das desigualdades sociais e a dirigir seus atos políticos para a transformação das condições mais globais.

\section{Conclusão}

Desde suas primeiras formulações no Brasil, a $\mathrm{RD}$ se opõe à política de guerra às drogas, que centraliza ações e programas na esfera do consumo de drogas ilícitas, propondo medidas repressivas e punitivas direcionadas aos consumidores e, na melhor das hipóteses, incentivando tratamento nos serviços de saúde com vistas à abstinência.

De maneira geral, no Brasil, a RD se apresenta como um conjunto de práticas de saúde fundamentadas na perspectiva teórica que concebe a existência de diferentes formas de relação do homem com as drogas, nem sempre vinculadas a um consumo problemático e, nesse sentido, seus objetivos não se restringem à eliminação do consumo. As medidas propostas visam a um conjunto de políticas voltadas para a redução de riscos e danos relacionados a todo tipo de consumo potencialmente prejudicial, distanciando-se do enfoque da guerra às drogas.

Ainda que se possa tomar essa formulação geral como ponto de partida, pode-se concluir, a partir da análise das publicações brasileiras, que a RD, como qualquer prática social, reflete as diferentes concepções em disputa nos campos dos saberes e 
das práticas de saúde e da produção, comércio e consumo de substâncias psicoativas, podendo-se falar concretamente na existência de várias RDs.

Os trabalhos analisados apontam diferentes concepções de objeto e de sujeito da $\mathrm{RD}$ apresentadas por diferentes autores. No entanto, a análise não permite apontar as diferenças entre os autores, pois há situaçōes que, em um mesmo trabalho ou em trabalhos diferentes de um mesmo autor, se observam concepções diversas.

A RD tomou inicialmente como objeto as doenças transmissíveis, especialmente a Aids, sendo a finalidade do trabalho a prevenção desses problemas entre usuários de droga injetável. Com o objeto assim circunscrito, atingiamse populações usuárias de drogas consideradas marginalizadas ou excluídas. As primeiras formulações teóricas que orientaram as práticas encontravamse entremeadas de críticas às abordagens e terapêuticas que unicamente perspectivavam a abstinência. Apesar da crítica por vezes contumaz aos modelos rígidos e intolerantes de combate às drogas, a finalidade de abstinência persiste nos discursos de uma parcela considerável daqueles que acabavam adotando, em maior ou menor grau, a orientação da RD.

Dada a complexidade e a envergadura que o fenômeno do consumo de drogas assumiu contemporaneamente, o que levou ao envolvimento de diversas áreas com esse objeto, o debate teórico em torno da RD se ampliou. A adoção de práticas de $\mathrm{RD}$, diante das crescentes contradições sociais e de saúde que envolvem o complexo sistema das drogas, colocou para a arena das discussões acadêmicas e dos serviços o tema da ética, dos direitos humanos, do engodo proibicionista, entre outros. A RD foi se constituindo então como um "movimento" político, que procurava dar respostas a essas contradições.

Dessa forma, a RD se ampliou, tornando-se objeto de vários campos do saber e acionando um debate mais acalorado na sociedade sobre os modos como as questôes relacionadas ao consumo de substâncias psicoativas deveriam ser encaminhadas. Nota-se que, além da saúde pública/epidemiologia e da psiquiatria, outras áreas do saber têm tomado a RD como objeto, como é o caso da psicologia, da saúde coletiva, das ciências sociais, da antropologia, do serviço social e da criminologia crítica, o que tem servido de base para ampliar seu alcance e fomentar o debate sobre as questóes que envolvem o complexo sistema das substâncias psicoativas. 
Sugere-se, neste trabalho, que a construção dos saberes e das respostas

sociais relativas ao complexo sistema das substâncias psicoativas vincule-se, através de categorias de análise totalizantes, aos processos globais da sociedade contemporânea e a suas contradições.

\section{Referências}

BARDIN, L. Análise de conteúdo. Lisboa: Edições 70, 2006.

ANDRADE, T.M. Redução de danos: mais que uma estratégia de prevenção entre UDIS. In: Atualidades em DST/Aids: redução de danos. São Paulo: Secretaria Estadual da Saúde, 1998. p. 89-93.

ANDRADE, T.M. et al. Redução de danos e redução da prevalência de infecção pelo HIV entre usuários de drogas injetáveis em Salvador-Bahia. In: A contribuição dos estudos multicêntricos frente à Epidemia de HIVIAids entre UDI no Brasil: 10 anos de pesquisa e redução de danos. Brasília: Ministério da Saúde, 2001. p. 95-114.

BASTOS, F.I. Por uma economia simbólica das trocas... de seringas. In: BASTOS, F.I.; MESQUITA, F.; MARQUES, L.F. (Org.). Troca de seringas: ciência, debate e saúde pública. Brasília: Ministério da Saúde, 1998. p. 91-100.

BASTOS, F.I. Redução de danos e saúde coletiva: reflexões a propósito das experiências internacional e brasileira. In: BASTOS F.; KARAM M.L.; MARTINS S.M. (Org.). Drogas, dignidade e inclusão social: a lei e a prática de redução de danos no Rio de Janeiro. Rio de Janeiro: ABORDA, 2003. p. 15-41.

BASTOS, F.I.; MESQUITA, F.; MARQUES, L.F. (Org.). Troca de seringas: ciência, debate e saúde pública. Brasília: Ministério da Saúde, 1998.

BERRIDGE, V. Harm minimisation and public health: Na historical perspective. In:

Psychoactive drugs and harm reduction: From faith to science. London: Whurr, 1993. p. 55-64.

BIRMAN, J. Arquivos do mal-estar e da resistência. Rio de Janeiro: Civilização Brasileira, 2006.

BRASIL. Ministério da Saúde. Manual de Redução de Danos. Brasília: Ministério da Saúde, 2001a.

BRASIL. Ministério da Saúde. Projeto ajude-Brasil: avaliação epidemiológica dos usuários de drogas injetáveis dos projetos de redução de danos. Brasília: Ministério da Saúde, 2001b.

BRASIL. Ministério da Saúde. A Política do Ministério da Saúde para Atenção Integral a Usuários de Álcool e outras Drogas. Brasília: Ministério da Saúde, 2004.

BRITES, C.M. Ética e uso de drogas: uma contribuição da ontologia social para o campo da saúde pública e da redução de danos. 2006. Tese (Doutorado em Serviço Social) - Pontifícia Universidade Católica de São Paulo, São Paulo, 2006. 
BUENO, R. Estratégias de redução de danos em Santos, SP. In: BASTOS, F.I.; MESQUITA, F.; MARQUES, L. F. (Org.). Troca de seringas: ciência, debate e saúde pública. Brasília: Ministério da Saúde, 1998. p. 163-169.

CALIPO, S.M. Saúde, Estado e Ética: NOB/96 e Lei das Organizações Sociais: a privatização da instituição pública da saúde? 2002. Dissertação (Mestrado em Enfermagem) - Escola de Enfermagem, Universidade de São Paulo, São Paulo, 2002.

CAMPOS, M.A., SIQUEIRA, D.J.R. Redução de danos e terapias de substituição em debate: contribuição da Associação Brasileira de Redutores de Danos. J. Bras. Psiquiatria, São Paulo, v. 52, n. 5, p. 387-393, 2003.

CANOLETTI, B.; SOARES, C.B. Programas de prevenção ao consumo de drogas no Brasil: uma análise da produção científica 1991 a 2001. Interface Comum Saúde Educação, São Paulo, v. 9, n. 16, p. 115-129, 2005.

CARLINI, E.A. Redução de danos: uma visão internacional. J. Bras. Psiquiatria, São Paulo, v. 52, n. 5, p. 335-339, 2003.

CARLINI-MARLATT, B.; REQUIÃO, D. H.; STACHON, A. C. Redução de danos: uma abordagem de saúde pública. J. Bras. Psiquiatria, São Paulo, v. 52, n. 5, p. 381-386, 2003.

CARNEIRO, H. As necessidades humanas e o proibicionismo das drogas no século XX. Rev. Out. IES, v. 6, p. 115-28, 2002.

CRUZ, M.S.; SÁAD, A.C.; FERREIRA, S.M.B. Posicionamento do Instituto de Psiquiatria da UFRJ sobre as estratégias de redução de danos na abordagem dos problemas relacionados ao uso indevido de álcool e outras drogas. J. Bras. Psiquiatria, São Paulo, v. 52, n. 5, p. 355-362, 2003.

DELGADO, P.G.; MACEDO, P.R.A.; CORDEIRO, F. et al. Álcool e redução de danos: construção de uma política intersetorial efetiva. In: MELCOP, A.G.; MAIA, D.; BUNING, E. et al (Org.). Relatório da I Conferência Internacional sobre Consumo de Álcool e Redução de Danos: em busca de uma política abrangente para os países em transição e desenvolvimento. Recife: Rede Brasileira de Redução de Danos, 2003. p. 127-40.

DIAS, J.C.; SCIVOLETTO, S.; SILVA, C.M. et al. Redução de danos: posições da Associação Brasileira de Psiquiatria e da Associação Brasileira para Estudos do Álcool e Outras Drogas. J. Bras. Psiquiatria, São Paulo, v. 52, n.5, p. 341-348, 2003.

FERNANDEZ, O.F.R.L. A prática de injeções de drogas, o uso comunitário de seringas e a redução dos riscos ao HIV. In: PARKER, R.; BASTOS, C.; GALVÃO, J. et al. (Org.). A Aids no Brasil: 1982-1992. Rio de Janeiro: ABIA, 1994. p. 252-69.

FERREIRA, V.R.T. Relato de uma experiência com adolescentes sobre o uso de drogas. Psicologia Teoria Prática, v. 5, n. 2, p. 47-54, 2003.

FIGUEIREDO, R.M.M.D.; GREDORI, R. Prevenção ao abuso de drogas em ações de saúde e educação: uma abordagem sócio-cultural e de redução de danos. São Paulo: NEPAIDS, 2002. 
FONSECA, E.M. Politicas de redução de danos ao uso de drogas: o contexto internacional e uma análise preliminar dos programas brasileiros. 2005. 112p. Dissertação (Mestrado em Saúde Pública) - Escola Nacional de Saúde Pública Sérgio Arouca, Fundação Oswaldo Cruz, Rio de Janeiro, 2005.

FRANCH, M. Um brinde à vida: reflexões sobre violência, juventude e redução de danos no Brasil. In: MELCOP, A.G.; MAIA, D.; BUNING, E. et al. (Org.). Relatório da I Conferência Internacional sobre Consumo de Álcool e Redução de Danos: em busca de uma política abrangente para os países em transição e desenvolvimento. Recife: Rede Brasileira de Redução de Danos, 2003. p. 49-71.

GORGULHO, M. O papel da mídia na promoção do uso responsável de álcool. In: MELCOP, A.G.; MAIA, D.; BUNING, E. et al. (Org.). Relatório da I Conferência Internacional sobre Consumo de Álcool e Redução de Danos: em busca de uma política abrangente para os países em transição e desenvolvimento. Recife: Rede Brasileira de Redução de Danos, 2003. p. 103-110.

GRANJEIRO, A.; CATAPANO, R.; EMILY, A. Redução de Danos. In: Atualidades em DSTIAIDS: redução de danos. São Paulo: Secretaria Estadual da Saúde, 1998. p. 7-39.

GUERRA, A.B.; MARQUES, A.C.P.R.; NOTO, A.R. et al. Posicionamento da Disciplina de Medicina e Sociologia do Abuso de Drogas. J. Bras Psiquiatria, São Paulo, v. 52, n. 5, p. 363-365, 2003.

HACKER, M.; TELLES, P.; BASTOS, F.I. Uma década de pesquisas sobre usuários de drogas injetáveis e HIV/Aids no Rio de Janeiro: parte I: rumo a uma epidemia sob controle? In: A contribuição dos estudos multicêntricos frente à Epidemia de HIVIAids entre UDI no Brasil: 10 anos de pesquisa e redução de danos. Brasília: Ministério da Saúde, 2001. p. 49-78.

KARAM, M.L. Redução de danos, ética e lei: os danos da política proibicionista e as alternativas compromissadas com a dignidade do indivíduo. In: BASTOS, F.; KARAM, M.L.; MARTINS, S.M. Drogas, dignidade e inclusão social: a lei e a prática de redução de danos no Rio de Janeiro. Rio de Janeiro: ABORDA, 2003. p. 45-97.

KRAL, A.; BUENO, R.; MESQUITA, F. Aids entre usuários de drogas injetáveis na região metropolitana de Santos, na década de 1990. In: A contribuição dos estudos multicêntricos frente à Epidemia de HIVIAids entre UDI no Brasil: 10 anos de pesquisa e redução de danos. Brasília: Ministério da Saúde, 2001. p. 11-48.

KUCHENBECKER, R.; SUDBRACK, M. Muito além da troca de seringas: a experiência do Programa de Redução de Danos associados ao uso de drogas injetáveis em Porto Alegre. Divulg. Saúde Debate, v. 24, p. 98-105, 2001.

LACAZ, F.A.C. O sujeito n(d)a saúde coletiva e pós-modernismo. Ciência Saúde Coletiva, v. 6, n. 1, p. 233-242, 2001.

MACRAE, E.; GORGULHO, M. Redução de danos e tratamento de substituição: posicionamento da Rede Brasileira de Redução de Danos. J. Bras. Psiquiatria, São Paulo, v. 52, n. 5, p. 371-374, 2003. 
MALBERGIER, A.; ANDRADE, A.G.; SCIVOLETTO, S. Redução de danos: Departamento e Instituto de Psiquiatria da Faculdade de Medicina da Universidade de São Paulo. J. Bras. Psiquiatria, São Paulo, v. 52, n. 5, p. 375-380, 2003.

MARQUES, F.; DONEDA, D. A política brasileira de redução de danos à saúde pelo uso indevido de drogas: diretrizes e seus desdobramentos nos Estados e Municípios. Mundo da Saúde, v. 23, n. 1, p. 10-9, 1999.

MARTINS, S.M. Norma, desvio e uso de drogas: o contexto da busca da cidadania pela redução de danos. In: BASTOS, F.; KARAM, M.L.; MARTINS, S.M. Drogas, dignidade e inclusão social: a lei e a prática de redução de danos no Rio de Janeiro. Rio de Janeiro: ABORDA, 2003. p. 101-14.

MELCOP, A.G.T. Vamos parar por aqui? Os desafios da abordagem de redução de danos nas violências no trânsito. In: MELCOP, A.G.; MAIA, D.; BUNING, E. et al. (Org.). Relatório da I Conferência Internacional sobre Consumo de Álcool e Redução de Danos: em busca de uma política abrangente para os países em transição e desenvolvimento. Recife: Rede Brasileira de Redução de Danos, 2003. p. 103-110.

MESQUITA, F. Dar oportunidade de vida ao usuário de drogas injetáveis: polêmica nacional. In: BASTOS, F.I.; MESQUITA, F.; MARQUES, L.F. (Org.). Troca de seringas: ciência, debate e saúde pública. Brasília: Ministério da Saúde, 1998. p. 101-112.

MESQUITA, F.; RIBEIRO, M.M. As estratégias de redução de danos junto a usuários de drogas injetáveis (a troca de seringas): aspectos médicos legais. In: Atualidades em DST/Aids: redução de danos. São Paulo: Secretaria Estadual da Saúde, 1998. p. 61-75.

MOREIRA, F.G.; SILVEIRA, D.X.; ANDREOLI, S.B. Redução de danos do uso indevido de drogas no contexto da escola promotora de saúde. Rio de Janeiro. Ciência Saúde Coletiva, v. 11, n. 3, p. 807-816, 2006.

MOREIRA, F.; SILVEIRA, D.X. Posicionamento do Programa de Orientação e Atendimento a Dependentes (PROAD). J. Bras Psiquiatria, São Paulo, v. 52, n. 5, p. 365-370, 2003.

NARDI, H.C.; RIGONI, R.Q. Marginalidade ou cidadania? A rede discursiva que configura o trabalho dos redutores de danos. Psicologia em Estudo, Maringá, v. 10, n. 2, p. 273-282, 2005. O'HARE, P. Redução de danos: alguns princípios e a ação prática. In: MESQUITA, F.; BASTOS, F.I. (Org.). Drogas e Aids: estratégias de redução de danos. São Paulo: Hucitec, 1994. p. 65-78.

PAES, P.C.D. Tá legal: construindo uma política pública de redução de danos no Estado de Mato Grosso do Sul, fronteira com a Bolívia. Campo Grande, Mato Grosso do Sul: GASS, 2002.

REALE, D. O caminho da redução de danos associados ao uso de drogas: do estigma à solidariedade. 1997. Dissertação (Mestrado em Medicina Preventiva) - Faculdade de Medicina, Universidade de São Paulo, São Paulo, 1997. 
RIBEIRO, M.; LARANJEIRA, R.R. Posicionamento da Unidade de Atendimento a Dependentes (UNIAD). J. Bras. Psiquiatria, São Paulo, v. 52, n. 5, p. 365-366, 2003.

RODRIGUES, T.M.R. Narcotráfico: uma guerra na guerra. São Paulo: Desatino, 2003.

SANTOS, V.E. O objeto/sujeito da redução de danos: uma análise da literatura da perspectiva da Saúde Coletiva. Dissertação (Mestrado em Enfermagem) - Escola de Enfermagem, Universidade de São Paulo, São Paulo, 2008.

SILVEIRA, C.; DONEDA, D.; GANDOlFI, D. et al. Política do Ministério da Saúde para atenção integral a usuários de álcool e outras drogas. J. Bras. Psiquiatria, v. 52, n. 5, p. 349-354, 2003.

SOARES, C.B. Adolescentes, drogas e AIDS: avaliando a prevenção e levantando necessidades. 1997. 294 p. Tese (Doutorado em Educação) - Faculdade de Educação, Universidade de São Paulo, São Paulo, 1997.

SOARES, C.B. Consumo contemporâneo de drogas e juventude: a construção do objeto na perspectiva da saúde coletiva. 2007. 207 p. Tese (Livre-docência em Enfermagem) - Escola de Enfermagem, Universidade de São Paulo, São Paulo, 2007.

SOARES, C.B.; CAMPOS, C.M.S. A responsabilidade da universidade pública no ensino da prevenção do uso prejudicial de drogas. Mundo Saúde, v. 28, n. 1, p. 110-115, 2004.

SODELLI, M. Aproximando sentidos: formação de professores, educação, drogas e açôes redutoras de vulnerabilidade. 2006. 250 p. Tese (Doutorado em Educação) - Psicologia da Educação, Pontifícia Universidade Católica de São Paulo, São Paulo, 2006.

STIMSOM, G.V. A Aids e o uso de drogas injetáveis no Reino Unido, 1987-1993: As políticas públicas e a prevenção da epidemia. In: BASTOS, F.I.; MESQUITA, F.; MARQUES, L.F. (Org.). Troca de seringas: ciência, debate e saúde pública. Brasília: Ministério da Saúde, 1998. p. 9-54.

STOTZ, E.N.; ARAÚJO, J.W.G. Promoção da saúde e cultura política: a reconstrução do consenso. Saúde Sociedade, v. 13, n. 2, p. 5-19, 2004.

STRONACH, B. Álcool e redução de danos. In: MELCOP, A.G.; MAIA, D.; BUNING, E. et al. (Org.). Relatório da I Conferência Internacional sobre Consumo de Álcool e Redução de Danos: em busca de uma política abrangente para os países em transição e desenvolvimento. Recife: Rede Brasileira de Redução de Danos, 2003. p. 29-35.

TAVARES-DE-LIMA, F.F. Prevenção ao uso de drogas: modelos utilizados na educação, suas relações e possibilidades quanto a atitudes preventivas. 2003. 115 p. Dissertação (Mestrado em Educação) - Psicologia da Educação, Pontifícia Universidade Católica de São Paulo, São Paulo, 2003.

TELLES, P.R.; BASTOS, F.I.; INCIARDI, J.A. Uma década de pesquisas sobre usuários de drogas injetáveis e HIV/Aids no Rio de Janeiro. Parte II: uma agenda para a ação, a experiência carioca. In: A contribuição dos estudos multicêntricos frente à Epidemia de HIVI 
Aids entre UDI no Brasil: 10 anos de pesquisa e redução de danos. Brasília: Ministério da Saúde, 2001. p. 79-94.

TRIGUEIROS, D.; BUENO, R. O projeto de redução de danos da cidade de São Paulo. In: SOUZA, C.R.; MESQUITA, F. (Org.). DST/Aids: a nova cara da luta contra a epidemia na cidade de São Paulo. São Paulo: Raiz da Terra, 2003. p. 39-45.

TRIVIÑOS, A.N.S. Introdução à pesquisa em ciências sociais: a pesquisa qualitativa em educação. São Paulo: Atlas, 1992.

VELOSO, L.; CARVALHO, J.; SANTIAGO, L. Redução dos danos decorrentes do uso de drogas: uma proposta educativa no âmbito das políticas públicas In: BRAVO, M.I.S.; VASCONCELOS, A.M.; GAMA, A.S. et al (Org.). Saúde e serviço social. Rio de Janeiro: Cortez, 2004. p 165-178.

WOOD, E.M. O que é a agenda "pós-moderna”? In: WOOD, E.M., FOSTER, L.B. (Org.). Em defesa da história: marxismo e pós-modernismo. Rio de Janeiro: Jorge Zahar, 1999. p. 13. 
Harm reduction: analysis of the concepts that guide practices in Brazil

This paper aimed to analyze the concepts that guide practices of Harm Reduction (HR) in Brazil. Forty six national publications were selected from 1994 to 2006, specially from LILACS database. From the central categories - object (what you want to transform) and subject (for whom the actions are directed), the analysis showed that the HR is very heterogeneous, with the unity object/subject represented by different equations: dependence-dependent; transmissible disease-drugusers of the marginalized and excluded populations; consumption-user of drugs; residents-ways of life of a particular "community", social risks and the general public; and production, trade and consumption of drugssocial class. It can be concluded that the different trends of HR have been built in Brazil as answers to problems related to the complex phenomenon of drug use, and mean the involvement of several areas and the extension of theoretical debate.

Key words: harm reduction, street drugs, qualitative analysis. 